\title{
The Effect of COVID-19 Illness on Pregnant Patients With Rheumatic Disease: Early Reassuring Data
}

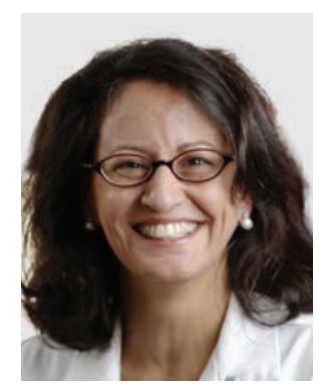

\author{
Lisa R. Sammaritano ${ }^{1}$
}

As of September 20, 2021, the World Health Organization (WHO) reported 228,206,384 cases of coronavirus disease 2019 (COVID-19), with over 4.5 million deaths worldwide. ${ }^{1}$ International responses by healthcare providers (HCPs), medical and pharmacologic researchers, and public health workers identified risk factors for serious illness and developed novel therapies and vaccines in real time, even as new variants emerge. Every HCP has been affected, even those not providing direct care to COVID-19-infected patients.

Rheumatologists have endeavored to characterize the intersection of COVID-19 with rheumatic diseases (RDs) as well as with immunosuppressive medications. To provide effective care and counsel to patients, we have all answered innumerable patient questions throughout the pandemic, our responses most often based on ongoing research from colleagues. One group, the COVID-19 Global Rheumatology Alliance (C19-GRA), was established within days of the WHO global pandemic declaration on March 11, 2020, and, along with other important research groups, has helped provide answers to some of these important questions.

Analysis of C19-GRA physician-reported registry data identified risk factors for hospitalization of COVID-19-infected patients with $\mathrm{RD}$, including prednisone $>10 \mathrm{mg}$ daily (OR 2.05, 95\% CI 1.06-3.96), and showed reduced odds of hospitalization with anti-tumor necrosis factor (TNF) agents (OR 0.40, 95\% CI $0.19-0.81){ }^{2}$ Increased risk of death was associated with older age, male sex, and specific comorbidities as identified in non-RD patients, but also rheumatology-specific factors of moderatehigh disease activity and certain medications. ${ }^{3}$ The death rate in the group of 3729 patients with RD was $10.5 \%,{ }^{3}$ with poorer COVID-19 outcomes in ethnic minorities in the US. ${ }^{4}$

${ }^{1}$ L.R. Sammaritano, MD, Professor, Weill Cornell Medicine, Hospital for Special Surgery, New York, New York, USA.

The author declares no conflicts of interest relevant to this article. Address correspondence to Dr. L.R. Sammaritano, Professor of Clinical Medicine, Weill Cornell Medicine, Hospital for Special Surgery, 535 East 70th Street, New York, NY 10021, USA. Email:sammaritanol@hss.edu.
Other authors have presented analyses of electronic medical record data that suggest RD patients with COVID-19 illness may be at higher risk of hospitalization, intensive care unit (ICU) admission, acute renal failure, and venous thromboembolism when compared to other patients with COVID-19. ${ }^{5}$ In contrast, a large systematic review and metaanalysis of 26 studies of COVID-19 in patients with RD (compared to the general population) suggested an OR of 1.53 for risk of COVID-19 illness (95\% CI 1.24-1.88); however, they did not find a higher risk of hospitalization and severe clinical outcomes. ${ }^{6}$ They did find a reduced risk associated with TNF inhibitor therapy use. ${ }^{6}$ Rheumatology medications clearly have the potential to affect COVID-19 infection and vaccination outcomes. ${ }^{7}$ Most rheumatology COVID-19 studies have focused on the broad issues of RD medication use, associated risk factors, and COVID-19 vaccines. In this issue of The Journal of Rheumatology, however, Bermas et $\mathrm{al}^{8}$ and the C19-GRA group report data on COVID-19 infection in an important and specific subset of RD patients with further altered physiology: pregnant patients. While numbers of pregnant patients with $\mathrm{RD}$ are small at any one time, the impact of infection in this complicated situation may be significant.

It is well recognized that underlying RD during pregnancy may increase risks to maternal and fetal/neonatal health. Pregnancy risk depends on underlying RD diagnosis, disease activity, presence of severe disease-related damage, current medications, and presence of relevant autoantibodies such as antiphospholipid and anti-Ro/La antibodies. ${ }^{9}$

The COVID-19 pandemic now introduces a new unknown into an already complex and incompletely understood situation. Pregnancy itself increases risk of some infections, with negative effects on maternal health and fetal/neonatal outcomes. Certain infections acquired in utero (or during the birth process) can cause fetal/neonatal mortality and childhood developmental anomalies. Classic TORCH infections are Toxoplasmosis, Other (syphilis), Rubella, Cytomegalovirus, and Herpes simplex virus. More recently recognized causes of intrauterine infection include enteroviruses, varicella zoster virus, parvovirus B19, and Zika virus.

\section{See COVID-19 in pregnant women, page 110}


In contrast to significant risk of intrauterine infection, corona and influenza viruses pose a greater threat to maternal health, rather than directly to fetal health. Previous coronavirus (Severe Acute Respiratory Syndrome [SARS] and Middle East Respiratory Syndrome [MERS]) and influenza A H1N1 outbreaks increased risk of maternal death and ICU admission as well as secondary adverse obstetric outcomes such as prematurity with little risk of vertical transmission. ${ }^{10}$

Studies of COVID-19 in pregnancy suggest risks similar to those seen with previous coronaviruses. Important risk factors for severe COVID-19 in pregnant women mirror those in the general population. There appears to be little risk of vertical transmission to the fetus, but the pregnant state increases risk of severe maternal infection. ${ }^{10} \mathrm{~A}$ recent study utilizing nonadmission-based standardized clinical criteria for severe disease found that pregnant women with symptomatic COVID-19 $(\mathrm{n}=22)$ were at significantly higher risk for severe infection, with adjusted relative risks, utilizing 2 different sets of criteria, of 3.59 (95\% CI 1.49-7.01) and 5.65 (95\% CI 1.36-17.31). ${ }^{11}$

Further, a "living" systematic review and metaanalysis of 192 studies found that pregnant women with COVID-19 evaluated at or admitted to hospitals were less likely to have typical symptoms of fever and dyspnea, but more likely to be admitted to the ICU or need mechanical ventilation. Comorbidities identified as risk factors for severe COVID-19 in pregnancy included non-White ethnicity, hypertension, diabetes, greater maternal age, and high BMI. COVID-19-infected pregnant patients were more likely to deliver preterm, and babies were more likely to be admitted to the neonatal ICU. ${ }^{12}$

In the current report by Bermas et al, the C19-GRA group reports data on $39 \mathrm{RD}$ patients with documented COVID-19 during pregnancy. ${ }^{8}$ Patients did well, with no deaths and only 2 of 39 requiring supplemental oxygen. Whereas one-quarter of patients were hospitalized, most patients did not receive specific COVID-19 medications.

Due to the registry methodology employed, the study lacks a true matched control group, making it difficult to know whether pregnancy itself increased risk of severe COVID-19 in these patients with $\mathrm{RD}$. The pregnant $\mathrm{RD}$ group as a whole appears to be at low risk for severe COVID-19 infection based on age, sex, disease activity, immunosuppressive medication use, and corticosteroid dose as compared to the general RD population reported in previous C19-GRA publications. Comorbidities were present in only 8 patients and corticosteroid use was reported in $23 \%$, with most doses being low ( $<5 \mathrm{mg}$ daily). In lieu of a control group, the authors compared outcomes in the pregnant group with those of all (nonpregnant) RD females between ages 20-45 years. The pregnant RD patients appeared to have a higher rate of hospitalization than did the nonpregnant patients, whether due to an excess of caution in the setting of pregnancy or to more significant symptoms. Overall hospitalization rate was $26 \%$ for pregnant $\mathrm{RD}$ patients vs $7 \%$ for nonpregnant $\mathrm{RD}$ comparison group. No pregnant patient died of COVID-19. The rate of death in the age-matched nonpregnant women was $2 \%$, and in the general registry was $10.5 \%{ }^{3}$

A registry dependent on voluntary reporting has poten- tial for data entry bias. Cases, entered by interested, motivated participating rheumatologists, may have skewed toward more complex disease or more severe outcomes. Another potential confounder was the use of RD medications that may have affected COVID-19 outcomes. While almost half of pregnant patients were on no rheumatology medications, 6 (15\%) were on anti-TNF therapies, which may be protective. Numbers are too small, however, to analyze effects of specific medications.

The authors suggest that differences in ethnic and racial makeup may play a role in differential outcomes of COVID-19 infection in pregnancy, as has been suggested in some series. ${ }^{13}$ Patients in this cohort were 33\% White, 23\% Latin American/ Hispanic, 23\% Arab or Middle Eastern, 15\% South or East Asian, and 5\% Black. ${ }^{8}$ Although the patients were entered from over 40 different countries, additional sociodemographic details were not reported.

This study reports on the largest number of pregnant RD patients with COVID-19 to date. Previous publications suggested similar good outcomes. Barbhaiya et al published their single-center online patient survey experience regarding patients with RD and pregnancy. ${ }^{14}$ There were 1547 women aged $18-50$ years who responded, of whom 61 were pregnant during the pandemic. Of these, 5 had self-reported COVID-19. The pregnant women did not report more severe COVID-19 symptoms. They had a shorter symptom duration, but a greater incidence of loss of smell and taste as compared to the 136 nonpregnant women of similar age with COVID-19 infection. ${ }^{14}$ Smeele et al reported 2 women with SLE and mild symptomatic COVID-19 during pregnancy. ${ }^{15}$ They were not treated, had no complications, and delivered healthy neonates at term.

While the emphasis in the current study was on maternal outcome ${ }^{8}$ limited pregnancy outcome data were available for 22 patients. No woman delivered due to COVID-19 infection. Most births were at term, with only 3 preterm births $(<37$ weeks); however, no details of neonatal outcome were available.

Many questions remain to be answered, including potential impact on short- and long-term neonatal outcomes. The timing of infection during pregnancy may be important, but most pregnancy studies published to date, including this one, have limited numbers of women who contracted COVID-19 in the first trimester (only 3 in this study). ${ }^{8}$

Despite an apparent higher rate of hospitalization, these patients did well. No patient required ICU or mechanical ventilation despite the added risk factor of pregnancy. These data are especially welcome since both RD patients and pregnant women are routinely excluded from clinical therapy or vaccine trials. This latest report from the C19-GRA provides limited but reassuring information for clinical rheumatologists seeking to counsel their patients with RD who are currently pregnant or are considering pregnancy during the COVID-19 pandemic.

\section{REFERENCES}

1. World Health Organization. WHO Coronavirus (COVID-19) Dashboard. [Internet. Accessed September 21, 2021.] Available from: https://covid19.who.int

2. Gianfrancesco M, Hyrich KL, Al-Adely S, Carmona L, Danila MI, Gossec L, et al; COVID-19 Global Rheumatology Alliance. 
Characteristics associated with hospitalization for COVID-19 in people with rheumatic disease: data from the COVID-19 Global Rheumatology Alliance physician-reported registry. Ann Rheum Dis 2020;79:859-66.

3. Strangfeld A, Schäfer M, Gianfrancesco MA, Lawson-Tovey S, Liew JW, Ljung L, et al; COVID-19 Global Rheumatology Alliance. Factors associated with COVID-19-related death in people with rheumatic diseases: results from the COVID-19 Global Rheumatology Alliance physician-reported registry. Ann Rheum Dis 2021;80:930-42.

4. Gianfrancesco MA, Leykina LA, Izadi Z, Taylor T, Sparks JA, Harrison C, et al; COVID-19 Global Rheumatology Alliance. Association of race and ethnicity with COVID-19 outcomes in rheumatic disease: data from the COVID-19 Global Rheumatology Alliance physician registry. Arthritis Rheum 2021;73:374-80.

5. D'Silva KM, Jorge A, Cohen A, McCormick N, Zhang Y, Wallace ZS, et al. COVID-19 outcomes in patients with systemic autoimmune rheumatic diseases compared to the general population: a US multicenter, comparative cohort study. Arthritis Rheum 2021;73:914-20.

6. Wang Q, Liu J, Shao R, Han X, Su C, Lu W. Risk and clinical outcomes of COVID-19 in patients with rheumatic diseases compared with the general population: a systematic review and meta-analysis. Rheumatol Int 2021;41:851-61.

7. D'Silva KM, Wallace ZS. COVID-19 and disease-modifying anti-rheumatic drugs. Cur Rheum Rep 2021;23:28.

8. Bermas BL, Gianfrancesco M, Tanner HL, Seet AM, Aguiar MC, Al Adhoubi NK, et al. COVID-19 in pregnant women with rheumatic disease: data from the COVID-19 Global Rheumatology Alliance. J Rheumatol 2022;49:110-14.

9. Maguire S, O'Shea F. Management of Pregnancy in Rheumatic Disease. EMJ Rheumatol. 2021;8:86-93.

10. Wastnedge EA, Reynolds RM, Van Boeckel SR, Stock SJ, Denison FC, Maybin JA, et al. Pregnancy and COVID-19. Physiol Rev 2021;101:303-18.

11. Oakes MC, Kernberg AS, Carter EB, Foeller ME, Palanisamy A, Raghuraman N, et al. Pregnancy as a risk factor for severe coronavirus disease 2019 using standardized clinical criteria. Am J Ob Gynecol MFM 2021;3:100319.

12. Allotey J, Stallings E, Bonet M, Yap M, Chatterjee S, Kew T, et al. Clinical manifestations, risk factors, and maternal and perinatal outcomes of coronavirus disease 2019 in pregnancy: living systematic review and meta-analysis. BMJ. 2020;370:m3320.

13. Emeruwa UN, Spiegelman J, Ona S, Kahe K, Miller RS, Fuchs KM, et al. Influence of race and ethnicity on severe acute respiratory syndrome coronavirus 2 (SARS-CoV-2) infection rates and clinical outcomes in pregnancy. Ob Gynecol 2020;136:1040-3.

14. Barbhaiya M, Stamm B, Vitone G, Frey MB, Jannat-Khah D, Levine $\mathrm{J}$, et al. Pregnancy and rheumatic disease: experience at a single center in New York City during the COVID-19 pandemic. Arthritis Care Res 2021;73:1004-12.

15. Smeele HT, Perez-Garcia LF, Grimminck K, Schoenmakers S, Mulders AG, Dolhain RJ. Systemic lupus erythematosus and COVID-19 during pregnancy. Lupus 2021;30:1188-91. 